nisse: Beim Vergleich verschiedener Elemente, etwa der Alkalien, untereinander läßt sich (wahrscheinlich wegen der Verschiedenheit der Elektronenhüllen) keine eindeutige Massenabhängigkeit feststellen (Abb. 1). Demgegenüber zeigt Abb. 2 ein Beispiel einer Messung mit den Ionen ${ }^{6} \mathrm{Li}$ und ${ }^{2} \mathrm{Li}$ an Molybdän; in diesem Falle ist $\gamma_{6} / \gamma_{7}=1,12$ innerhalb der Meßgenauigkeit unabhängig von der Ionenenergie. Aus einer größeren Anzahl ähnlicher Messungen an verschiedenen, zum Teil ausgeheizten Metallen $(\mathrm{Cu}, \mathrm{Mo}, \mathrm{Pt}, \mathrm{Be}, \mathrm{Ni}, \mathrm{Cr}-\mathrm{Ni})$ und bei $\mathrm{Be}-$ schleunigungsspannungen zwischen 200 und 7000 Volt ergeben sich Werte für $\gamma_{6} / \gamma_{7}$ etwa zwischen 1,1 und 1,2. Die Unterschiede in den $\gamma_{6} / \gamma_{7}$-Werten sind nicht dem Meßfehler zuzuschreiben, sondern rühren offenbar von der Verschiedenheit der Oberflächen her. Mit den Kaliumisctopen 39 und 41 wurde der Wert $\gamma_{39} / \gamma_{41}=1,03 \pm 0,01$ gemessen.

Für das Verhältnis der Ionenreflexionsfaktoren wurden bei den Li-Isotopen Werte zwischen 1,1 und 1,25 becbachtet.

Eine ausführlichere Darstellung und Diskussion der Ergebnisse soll demnächst an anderer Stelle folgen.

\section{Zur Theorie der wandstabilisierten Bogensäule}

$$
\text { Von Georg } \mathrm{S} \text { chmitz* }
$$

(Z. Naturforschg. 5 a, 571 [1950]; eingeg. am 25. Sept. 1950)

Die Energiebilanz eines wandstabilisierten Lichtbogens, die im stationären Fall die zur Wand abgeleitete Wärmeleistung gleich der um die Abstrahlung verminderte Stromleistung setzt, führt zu einer nichtlinearen Differentialgleichung 2. Ordnung, die auch die 1. Ableitung der abhängigen Veränderlichen enthält und die die Temperatur als Funktion des Abstandes von der Entladungsachse festlegt 1,2 .

$\mathrm{Zu}$ einer etwas einfacheren Differentialgleichung gelangt man jedoch, wenn man zunächst die zur Wand abfließende Wärmeenergie $\boldsymbol{J}$ zum Gegenstand der Untersuchung macht. Für sie hat man im Abstand $r$ von der Achse den Ausdruck

$$
J=-2 . \tau r \% \frac{d T}{d r}
$$

( $\varkappa$ Wärmeleitvermögen, $T$ Temperatur).

* Troisdorf-Oberlar, Kirchstr. 19.

1 G. H ell e r, Physics 6, 389 [1935].

2 W. Weizel u. G. Ecker, Z. Physik 127, 495 [1950].
Da sich immer eine Funktion $G(T)$ so bestimmen läßt, daß

$$
\frac{d G}{d r}=\% \frac{d T}{d r}
$$

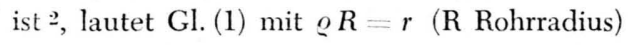

$$
J=-2 . \pi \varrho \frac{d G}{d \varrho},
$$

und mit $z=\ln \varrho$ ergibt sich für den Wärmestrom die Größe

$$
J=-2 . \tau \frac{d G}{d z}
$$

Im Innern der Bogensäule lautet damit für ein ringförmiges Volumenelement von der Dicke $r_{1}-r_{2}=d r$ die Energiebilanz

bzw.

$$
-2 \pi\left[\left(\frac{d G}{d z}\right)_{1}-\left(\frac{d G}{d z}\right)_{2}\right] \approx 2 \pi R^{2} \varrho(ङ i-s) d \varrho
$$

$$
-\frac{d^{2} G}{d z^{2}}=R^{2} \varrho^{2}(ङ i-s),
$$

die $G(T)$ als Funktion von $z$ bestimmt. ([5 Feldstärke, $i$ Stromdichte, $s$ Strahlungsdichte.)

Wesentlich ist, daß in Gl. (4) die 1. Ableitung der abhängigen Veränderlichen nicht mehr vorkommt, wodurch bei numerischen Integrationen der instrumentelle bzw. zeitliche Aufwand klein bleibt. Zudem geht die Lösungskurve bei größeren $z$-Werten, da in der Nähe der Rohrwand im allgemeinen kein Strom fließt und kein Licht entsteht, in eine Gerade über.

\section{Nachtrag}

zu der Notiz von K. Clusius: Bemerkung $\mathrm{zum} \mathrm{B}$ ewegung $\mathrm{s}$ e chan is mus der a ufsteigenden $\mathrm{H}_{2}-\mathrm{O}_{2}$ - D iff us ionsflammen*

Herr Prof. Ackeret von der E.T.H.Zürich machte mich freundlichst darauf aufmerksam, daß der Fall einer aufsteigenden Luftblase im flüssigkeitsgefüllten Rohr bereits von D. T. D u m it r e s c u (Z. angew. Math. Mechanik 23, 139 [1943]) behandelt worden ist. Dieser Autor findet experimentell und theoretisch in guter Übereinstimmung mit Davies und $\mathrm{T}$ a y lor die Steiggeschwindigkeit $\bar{z} u v_{s}=0,49 \sqrt{g a}$.

K. C l u s i us, Zürich.

* Z. Naturforschg. 5a, 514 [1950].

\title{
BESPRECHUNGEN
}

Natural Philosophy of Cause and Chance. Von $\mathrm{M} \mathrm{ax}$ B orn. Vorlesungen gehalten 1948 am St. Mary Magdalen College in Oxford. Clarendon Press, Oxford 1949, Preis geb. 17 s. 6 d.

Dieses Buch sei dem deutschen Leser aufs wärmste empfohlen. Der erste, mehr philosophisch gehaltene Teil desselben arbeitet, bei sparsamer Benutzung der Formelsprache, den Gegensatz zwischen der kausalen klassischen
Physik und der wahrscheinlichkeitstheoretischen modernen Physik heraus. Der zweite Teil, der aus 35 Appendices besteht und nicht viel kürzer ist als der erste, bringt originelle Neuheiten. Das ganze Buch zeigt den Verf. auf der Höhe der modernen Forschung, die er durch seine Edinburger Schule erfolgreich vorangetrieben hat.

Gelegentlich einer kurzen Analyse der Relativitätstheorie bespricht Verf. das Unbefriedigende von Einsteins 